\title{
BMJ Open Serological surveillance of influenza in an English sentinel network: pilot study protocol
}

\author{
Simon de Lusignan, ${ }^{1,2}$ Ray Borrow, ${ }^{3}$ Manasa Tripathy, ${ }^{1}$ Ezra Linley, ${ }^{3}$ \\ Maria Zambon, ${ }^{4}$ Katja Hoschler, ${ }^{4}$ Filipa Ferreira, ${ }^{1}$ Nick Andrews, ${ }^{5}$ Ivelina Yonova, ${ }^{1,2}$ \\ Mariya Hriskova, ${ }^{1,2}$ Imran Rafi, ${ }^{2}$ Richard Pebody ${ }^{6}$
}

To cite: de Lusignan $\mathrm{S}$, Borrow R, Tripathy M, et al. Serological surveillance of influenza in an English sentinel network: pilot study protocol. BMJ Open 2019;9:e024285. doi:10.1136/ bmjopen-2018-024285

- Prepublication history for this paper is available online. To view these files, please visit the journal online (http://dx.doi. org/10.1136/bmjopen-2018024285).

Received 18 May 2018 Revised 11 January 2019 Accepted 17 January 2019

Check for updates

(c) Author(s) (or their employer(s)) 2019. Re-use permitted under CC BY-NC. No commercial re-use. See rights and permissions. Published by BMJ.

${ }^{1}$ Department of Clinical and Experimental Medicine, University of Surrey, Guildford, UK

${ }^{2}$ Clinical Innovation and Research Centre (CIRC), Royal College of General Practitioners, London, UK

${ }^{3}$ Vaccine Evaluation Unit, Manchester Royal Infirmary, Public Health England, Manchester, UK

${ }^{4}$ Public Health England, London, UK

${ }^{5}$ Modelling and Economics Department, Public Health England, London, UK

${ }^{6}$ Centre for Infectious Disease Surveillance and Control, Public Health England, London, UK

Correspondence to

Professor Simon de Lusignan;

s.lusignan@surrey.ac.uk

\section{ABSTRACT}

Background Rapidly undertaken age-stratified serology studies can produce valuable data about a new emerging infection including background population immunity and seroincidence during an influenza pandemic. Traditionally seroepidemiology studies have used surplus laboratory sera with little or no clinical information or have been expensive detailed population based studies. We propose collecting population based sera from the Royal College of General Practitioners (RCGP) Research and Surveillance Centre (RSC), a sentinel network with extensive clinical data.

Aim To pilot a mechanism to undertake population based surveys that collect serological specimens and associated patient data to measure seropositivity and seroincidence due to seasonal influenza, and create a population based serology bank.

Methods and analysis Setting and Participants: We will recruit 6 RCGP RSC practices already taking nasopharyngeal virology swabs. Patients who attend a scheduled blood test will be consented to donate additional blood samples. Approximately 100-150 blood samples will be collected from each of the following age bands - 18-29, 30-39, 40-49, 50-59, 60- 69 and 70+ years.

Methods We will send the samples to the Public Health England (PHE) Seroepidemiology Unit for processing and storage. These samples will be tested for influenza antibodies, using haemagglutination inhibition assays. Serology results will be pseudonymised, sent to the RCGP RSC and combined using existing processes at the RCGP RSC secure hub. The influenza seroprevalence results from the RCGP cohort will be compared against those from the annual PHE influenza residual serosurvey.

Ethics and dissemination Ethical approval was granted by the Proportionate Review Sub- Committee of the London - Camden \& Kings Cross on 6 February 2018. This study received approval from Health Research Authority on 7 February 2018. On completion the results will be made available via peer-reviewed journals.

\section{INTRODUCTION}

Rationale for the pilot study and background There have been calls for a World Serology Bank as serology could tell us much about the susceptibility of the population to infectious

\section{Strengths and limitations of this study}

- The RCGP RSC is one of the oldest sentinel networks in Europe, it has completed its 51st season of surveillance.

- Practices in RCGP take microbiological samples, including influenza virology specimens which are linked at individual level.

- We have the potential through this network to link vaccine exposure and serology.

- This is a pilot study to demonstrate we can collect samples across predefined adult age-groups - we will not collect samples from children, who may be exposed to live attenuated intranasal vaccine (LAIV) in recent years.

- Pharmacist and other non-GP vaccination can lead to missing information on the computerised medical record system at General Practice.

disease. ${ }^{1}$ This pilot study explores the potential to establish a serology bank, based on a sentinel network, and focused on influenza.

Serological data potentially allow the assessment of the severity of a new influenza strain by providing the capability to detect asymptomatic and mild infections and thus determine the symptomatic proportion. ${ }^{2}$ The number of infections can be determined if the age-specific prevalence of immunity prior to and then during and after the pandemic are known. Thus, the number of people infected (and therefore no longer susceptible) can be calculated. If these data are available early (particularly the background population immunity and the symptomatic proportion), they can be used to adjust planning assumptions and to help predict the impact of the pandemic on healthcare services and optimal intervention strategies.

Serum archives would help assess the severity of a novel influenza virus and allow modification of local, national and international pandemic plans. ${ }^{3}$ This requirement was a lesson from the $2009 \mathrm{~A}(\mathrm{H} 1 \mathrm{~N} 1)$ pandemic 
and its importance is recognised by the WHO, the European Centre for Disease Control (ECDC), the UK Department of Health, and the Joint Committee on Vaccination and Immunisation.

Despite its apparent merit, the use of serology for seasonal influenza surveillance has several limitations. Seasonal influenza infections occur regularly and individuals are reinfected throughout life with related strains. The prior (lifetime) exposure of individuals to circulating influenza viruses in previous years and a high degree of cross-reactivity between antibodies to different seasonal influenza virus strains can lead to results which are difficult to interpret. Distinguishing between recent infection and recent vaccination also presents further difficulties. Consequently, seroepidemiological studies have not often been used for the investigation of seasonal influenza at a population level.

The UK undertook a series of influenza serosurveys during the 2009 pandemic based on residual blood samples from the Public Health England (PHE) National Seroepidemiology Programme. ${ }^{4}$ These are samples submitted to PHE and National Health Service (NHS) laboratories for routine diagnostic purposes. Although this work delivered critical information on background population seroprevalence and seroincidence, several issues were raised in postpandemic reviews. These reviews highlighted that, although this information was gathered and published earlier than almost any other country, even earlier availability of this intelligence would have been critical to inform important national policy decisions. The following key recommendations have been made in relation to influenza seroepidemiology.

- The Science and Technology Committee (third report 2010-2012) stated that seroepidemiological data need to be available earlier in the time course of a future pandemic to help with risk assessment (ie, the likely spread of the disease across the population). ${ }^{5}$

- The Chief Medical Officer-Statistics Legacy Group determined that serosurveillance is critical to determine population immunity and community infection rates. These data cannot be obtained from other sources and are vital to making modelling predictions of the pandemic. ${ }^{6}$

- The 2011 UK Influenza Pandemic Preparedness Strategy includes seroepidemiology as a key surveillance initiative that will be required at the start of any pandemic, and states that work should be underway to enhance capability to respond, based on the H1N1 (2009) influenza pandemic. ${ }^{7}$

- The Scientific Pandemic Influenza Advisory Committee (SPI), Subgroup on Modelling (SPI-M) group recommended that the PHE strengthen population based influenza seroepidemiology, including collection of key epidemiological information on vaccination status and underlying risk status. ${ }^{8}$

- Finally ECDC has highlighted the importance of influenza seroepidemiology, as has WHO as part of the Fineberg report into the pandemic response and the lack of preparedness including the need for a proper assessment of severity at national and subnational levels early in a pandemic. ${ }^{9}$

The Royal College of General Practitioners (RCGP) Research and Surveillance Centre (RSC) and PHE, and its predecessor organisations have an over 50 -year history of collaboration in influenza and respiratory disease surveillance and vaccine effectiveness studies. ${ }^{10}$ This study builds on this long-term collaboration.

The RCGP RSC provides a suitable venue for collecting influenza serology data. This sentinel network is one of the longest established and has a nationally representative network of practices. ${ }^{11}$ The network has a long history of feedback about data quality, particularly in the areas of influenza-like illness (ILI) and other respiratory infections. ${ }^{12}$ The RCGP RSC also collects data about vaccine exposure. Combining information about ILI, virology results and vaccine exposure enables the estimation of influenza vaccine effectiveness. ${ }^{13}$ Its dashboard capability provides a method for near real time feedback to practices about sample collection. ${ }^{14}$

This pilot study tests whether collecting serology data from a sentinel network and linking it to clinical record data at the individual level would provide a low cost way of creating a high quality seroepidemiological resource. ${ }^{15}$

\section{Aim}

To pilot a mechanism to undertake population based surveys that collect serological specimens that will be linked with key epidemiological information at strategic time points after each influenza season as a resource to be deployed in a future pandemic, for seasonal influenza and potentially other infections of public health importance.

\section{Objectives}

- Establish a system that allows volunteer patients from practices to provide a serology sample during a routine blood test. The result of that sample will be linked to that patient's pseudonymised record which will provide high quality data about vaccine exposure, and any medically reported ILI or other condition of scientific interest, included within an approved scientific protocol, or meeting public health needs in a pandemic.

- Measure the feasibility of collecting 100-150 samples from each of the following age-bands - 18-29, 30-39, $40-49,50-59,60-69$ and $70+$ years.

- Pilot laboratory programme for the processing and storage of these specimens.

- Pilot linkage of these biological specimens to the PHE Respiratory Virus Unit (RVU) analysis programme for the detection of influenza antibodies.

- Link the results to epidemiological data in particular vaccination history, age and underlying clinical risk factor status and any swab results. The serology results can be linked to vaccine brand. 
- Estimate the costs of providing a national seroepidemiology service based on samples from a subset of RCGP RSC practices.

\section{METHODS AND ANALYSIS \\ Study design}

Study setting and population

The project will consist of four interlinked work packages:

1. Population sampling and collection of biological specimens.

2. Laboratory analysis.

3. Data management.

4. Statistical and modelling analysis.

Population sampling and collection of biological specimens The RCGP RSC practices will collect the biological specimens.

The RCGP RSC will be used to collect the biological specimens. Practices who participated in the virology swabbing scheme will be invited.

The project proposes to pilot a population based seroprevalence survey, involving 100-150 individuals across the following age range: 18-29, 30-39, 40-49, 50-59, 60-69 and 70+ years, following the 2017/2018 influenza season. Patients, who attend their pilot sentinel network practice for routine blood test during the study period, will be asked to also provide an additional blood sample for serology. This will provide information on seroprevalence to set in context other measures of impact of an influenza season/epidemic in a population, and provide the most accurate measures of population exposure. This new approach to serology banking represents a compromise between using residual serum samples from laboratories where less is known about the patient's medical and immunisation history and formal surveys that can collect such data, but can have non-response bias.

Analysis will be carried out using the blood collected in RCGP RSC practices and sent to the Seroepidemiology Unit (SEU) archive. The SEU archive is a collection of anonymised residual serum samples from routine microbiological testing, submitted voluntarily each year from laboratories throughout England. SEU archive sera are stored at the PHE North West regional laboratory in Manchester and are anonymised and permanently unlinked from any patient identifying information, with only age, gender, date of collection (if available) and contributing laboratory retained.

Serology samples will be analysed at the RVU at the PHE Colindale using HAI with representative vaccine strains. Each sample will be tested once and not in duplicate, the result will have strong identifiers pseudonymised (in accordance with current best practice) and be returned to the RCGP RSC hub's encrypted server, decrypted and linked to individual patients pseudonymised data.

The RCGP RSC will recruit six practices (depending on practice list size) to ascertain the feasibility and establish the approach. We will aim to collect an evenly distributed set of sera across all age bands that are set out in table 1. Older people have more chronic disease and hence have higher number of blood tests performed than younger people; so the target number of samples would be achieved sooner. There will be no attempt to select patients for serology on the basis of whether they have had an influenza immunisation, or not or had a virology specimen taken for influenza. The number of samples is based on a combined list size of 50000 across the participating practices.

The proposed method would involve feeding back to practices as each age band reaches the target number of samples (minimum 100, maximum 150 per age band). University of Surrey has developed methods for RCGP RSC to give practice specific feedback about vaccine exposure and data quality - these methods could be used to provide feedback to practices participating in serum collection.

\section{Patient selection}

\section{Inclusion criteria}

All patients of age 18 and over who visit their practice for a routine blood test and provide another sample for serology are eligible for inclusion in the analysis. The

Table 1 Blood test plan for the pilot study

\begin{tabular}{lll}
\hline & \multicolumn{2}{l}{ All practices (5-6 practices) during the study period } \\
\cline { 2 - 3 } Age band & $\begin{array}{l}\text { 100 sample threshold crossed after the } \\
\text { following number of weeks }\end{array}$ & $\begin{array}{l}\mathbf{1 5 0} \text { sample threshold crossed after the } \\
\text { following number of weeks }\end{array}$ \\
\hline $18-29$ & 3 & 5 \\
$30-39$ & 3 & 5 \\
$40-49$ & 2 & 2 \\
$50-59$ & 1 & 2 \\
$60-69$ & 1 & 2 \\
$70+$ & 1 & 1 \\
\hline
\end{tabular}

This table represents the week in which sample collection would be complete, if all patients consented. The pilot study will take place as soon as ethical approval is achieved and would involve the collection of a total of up to 150 samples from approximately six practices over the study period. 
main inclusion criterion for practices is that practices are within our influenza swabbing practices' list with quota sampling according to the table above.

\section{Exclusion criteria}

Patients who have explicitly opted out of data sharing will be excluded from the analysis. We will identify these patients using the opt-out codes within General Practice (GP) information systems where the patients have made an explicit choice to opt out; patients will be informed of their option to opt-out via posters in the practices and information sheets.

\section{Laboratory analysis}

Samples will be submitted to the PHE Manchester laboratory in practice batches of clotted vacutainer bottles, and will be accompanied by the standard request form. This form will be generated by existing ICE pathology request software used by the GP practices.

General Practices use a pathology request software, such as the ICE system to send biological specimens to their local laboratory for testing. When a patient requires a blood test, the GP can print a test request form via their ICE system. To send samples to PHE's SEU/VEU:

- The practice will need to print out an additional test request form for routine blood sampling.

- The University of Surrey will provide the practices with detailed guidance on sample collection and postage to PHE's SEU/VEU.

On receiving consent, practices will be able to send specimens to PHE's SEU/VEU lab via pre-paid envelopes. A Material Transfer Agreement (MTA) will be put in place for the transport of blood samples from GP practices to Manchester SEU/VEU.

We suggest a single Read $\operatorname{code}^{16}$ is allocated to mark that the serology specimen has been consented to and sent. This will facilitate one-to-one matching of specimens with the consenting patient/subjects clinical history.

Whole blood samples will be received, processed to obtain sera, catalogued and stored in $-80^{\circ} \mathrm{C}$ archive freezers within the Serum Archives section of the Vaccine Evaluation Unit (VEU) at Manchester according to existing SOPs, modified where necessary. It is envisioned that sera collected will be permanently transferred to the SEU archive once this study is complete.

Sera will be processed to undergo analysis at the RVU of the National Infection Service of PHE (London, UK) using haemagglutination inhibition (HAI) assays and other measures of functional antibody status according to established protocols to detect antibody levels against relevant circulating and/or vaccine influenza strains. ${ }^{17}$ Antigens will be grown in-house in egg and cell-culture and Influenza B antigens will be diethyl ether extracted as previously described.$^{18}$ Briefly, for the HAI assay, sera will be treated to remove non-specific inhibitors using receptor destroying enzyme (RDEII) and then twofold serially diluted starting at a 1:10 dilution, followed by mixing with an equal volume $(25 \mu \mathrm{l})$ of PBS containing
Table 2 Read codes to flag that a specimen has been taken

\begin{tabular}{ll}
\hline V2_READ_CODE & V2_TERM \\
\hline 4JDb. & Influenza (A\&B) serology \\
$43 \mathrm{~L}$. & Sample serology \\
\hline
\end{tabular}

4HA units of each of the strains. Turkey red blood cells (RBC) will be used for the influenza $\mathrm{A}(\mathrm{H} 1 \mathrm{~N} 1)$ pdm09 and influenza B components, and Guinea pig RBC for H3N2. HAI titres will be expressed as the reciprocal of the last serum dilution that results in complete inhibition of agglutination. Where sample volumes after completion of the HAI permit, additional analysis using influenza neutralisation and/or Neuraminidase Antibody Inhibition assays in the format of the Enzyme-linked Lectin Assay ${ }^{16}$ may be considered to investigate the functionality of the antibody further.

Following analysis, the results will be reported in form of an Excel table (containing titres against each antigen/ influenza virus for every sample) to the data manager of the project. The data (with pseudonymised strong identifiers) will be linked to RCGP RSC data to allow analysis of relevant data including vaccine exposure and previous influenza.

\section{Using the UK laboratory bounded code list}

UK laboratories are currently obligated to use the Pathology Bounded Code List (PBCL), a subset of NHS Digital Read codes, when electronically reporting pathology results to GPs, but are freely allowed to choose which PBCL codes are used for each test. A PBCL code assigned to the test (table 2) will allow 1 week in areas for the RCGP RSC team to follow the collection of the samples by age band.

\section{Data extraction and data management}

\section{Data collection from volunteer RCGP RSC practice}

Data will be extracted from RCGP RSC databases which store pseudonymised data received from participating RCGP practices. ${ }^{10} \mathrm{~A}$ UK general practice is a registration based system where all citizens can register with a single GP of their choice. Practices are computerised, and data entered into computerised medical record systems either as coded data, or free text.

Practices will code 43L. (Sample serology) into patient record when a patient consents to providing a sample for Serology. We will extract the coded data, and our results will be based on this element of the record. We will extract all coded data, pseudonymising as close to sources as possible. Where patients have a range of codes inserted in their record suggesting they opt out of record sharing we will not analyse their data.

The RCGP RSC will only extract coded data, for example, where the GP or other health professional codes a disease or symptom into their computerised medical record system, and will process these data as required for 
this project. ${ }^{17}{ }^{19}$ Coded routine data from UK primary care has been widely used in research. ${ }^{20}$

\section{Data collection methods will follow best practice, including pseudonymisation}

These data extractions will be conducted in accordance with best practice, using the Clinical Informatics and Health Outcomes Research Group's standard operating procedures for data extraction, pseudonymisation, and transfer. The method and governance procedure has been developed by the University of Surrey, using an approved provider.

Pseudonymisation is the standard approach for protecting patient's privacy. It is a process that involves the removal of all personal identifiers from data - such as name, date of birth, etc. However, there is a risk that if data are linked to other data a person might be identified. ${ }^{21}{ }^{22}$ Therefore although all identifiers are removed we keep data encrypted during transfer and on a secure network that meets NHS Information Governance standards to minimise the risk of re-identification. A legally binding definition of pseudonymisation has been introduced into European law on the recommendation of the European Data Protection Supervisor (EDPS).$^{19}$

We 'pseudonymise' strong identifiers (in this study NHS number) so that we can link further data to the same individual's record. For this study we need, for example, to be able to link whether there have been any immune changes to the individual that had been vaccinated (and with the specific brand and batch number). Pseudonymisation allows us to do this without knowing any of the strong personal identifiers of that individual.

All data processing and analysis in the present proposed study will be conducted within the secure IT environment of the Clinical Informatics and Health Outcomes Research Group, at the University of Surrey. The information security policies and procedures of the Research Group have been approved by the NHS Digital as meeting the Information Governance Toolkit (IGT) standards. ${ }^{23}$

The following routinely collected patient data will be collected for the study:

- Demographic information: age, gender, ethnicity, registered date.

- Lower Super Output Area (LSOA): full postcodes will be automatically and immediately transformed into Lower Super Output Area (LSOA) which can be used for calculating deprivation scores, using the Index of Multiple Deprivation (IMD), within GP computer systems on extraction. This would provide information about any inequities in access according to level of social deprivation using geographical information system (GIS) methods.

- Influenza vaccination - including date of vaccination and brand/lot

- Primary care consultations following vaccination, any other markers of healthcare utilisation, and referral to further care.
- Reactogenicity outcomes of seasonal influenza vaccination as listed in the research literature and any contemporary EU guidance.

- Life-style/risk factors - for example, Body Mass Index (BMI), smoking status.

- Records of other diseases and long term conditions - for example, chronic respiratory disease, chronic heart disease, chronic kidney disease, chronic liver disease, chronic neurological disease, diabetes, immunosuppression, pneumonia, etc.

- Pregnancy.

- PHE results of influenza HAI testing will be linked to this data-set at the University of Surrey according to established information governance (IG) procedures

Practices will receive weekly feedback via our dashboard system about progress to target within each age band (table 1$)^{15}$

\section{Data tables for analysis}

RCGP RSC will prepare tables for analysis as set out in the section below on statistical modelling and analysis. A schedule for reports would be created, as is currently carried out for reporting influenza vaccine effectiveness studies, and blank tables prepared in advance as outlined in this protocol.

\section{Statistical and modelling analysis}

PHE will lead on this analysis and follow the outline plan set out below.

The proof-of-concept study will consist of a total of up to $100-150$ participants per age band from whom blood samples have been taken during the survey period.

The evaluation will include the following elements:

- A comparison of representativeness of the recruited study population will be undertaken in relation to national surveys.

- A comparison of the influenza seroprevalence results from the RCGP cohort against those from the annual PHE influenza residual serosurvey

- Demographic, epidemiological and lab data will be checked for completeness, errors and inconsistencies

- Samples with titres $>40$ by HAI will be considered seropositive to report prevalence of influenza antibody detected by HAI. ${ }^{4}$ In addition, geometric mean titres and reverse cumulative distribution curves will be calculated for some preliminary, exploratory comparisons of groups such as those vaccinated versus unvaccinated - although conclusions will be limited by the samples available.

- Acceptability to participate among practices and patients ascertained through measures such as response rate.

- Feasibility of extending the pilot approach to a wider number of practices.

University of Surrey will report the extent to which we have samples from common households - this might provide information about shared infections/levels of immunity within households. 
We will produce a weekly report on progress to target by age band and provide feedback to practices about the utility of the data provided. This will be a simple table based on table 1, explaining the anticipated number of weeks to complete the sampling by age band.

\section{Patient and public involvement}

No patients or public were involved in the development of the research question or design of this study.

\section{Project management}

The pilot project will be a collaborative project lead by Professor Simon de Lusignan at University of Surrey, with RCGP and PHE as collaborators. The RCGP-PHE scientific committee will oversee the project in collaboration with the principal investigator.

This research and information governance framework for RCGP RSC sits within the University of Surrey's formal frameworks for information and research governance. In addition, all externally funded projects and collaborative projects with external partners are supported and guided by the University's Research and Enterprise Support (RES) service. RES ensures that university-supported projects are financially viable, and that legal issues of knowledge transfer and intellectual properties are addressed. The project team is supported by IT services dedicated to the Faculty and to the Department of Clinical and Experimental Medicine. Our secure analysis servers are optimised for routine healthcare data processing, to provide faster deliveries for our projects.

\section{ETHICS AND DISSEMINATION}

\section{Ethical Approval}

PHE has ethical approval $(05 / \mathrm{Q} 0505 / 45)$ for the collection and use of unlinked and anonymised residual serum samples in cross-sectional antibody prevalence studies for the surveillance of population immunity to vaccine preventable diseases of public health importance and the collection has been extensively used for this purpose.

We will seek to collect serum samples from a cohort of patients that are registered with one of the RCGP RSC practices. Potential participants will be attending a pre-scheduled blood appointment, where the healthcare professional treating them will inform them about the study and seek their consent about whether they would be interested in donating an additional blood sample as part of the study.

Ethical approval was granted by the Proportionate Review Sub-Committee of the London - Camden \& Kings Cross on 6 February 2018. This study received approval from Health Research Authority on 7 February 2018.

Blood serum is acellular and not considered a material subject to the Human Tissue Act $2004 .{ }^{24}$ However, practices will all need to put in place a MTA with PHE prior to starting their surveillance. ${ }^{25}$

\section{Information governance}

The Clinical Informatics and Health Outcomes Research Group at the University of Surrey has worked with routinely collected healthcare data in a number of research and evaluation projects for over 20 years. ${ }^{26}$ The Research Group works within the research and Information Governance frameworks for health and social care in the United Kingdom, and is compliant with the University's best practice standards. The University of Surrey is registered with the Information Commissioner's Office Data Protection Register, and is compliant with the Data Protection Act, and other legislations.

In addition, the Research Group reviewed its departmental information governance policies and procedures, against the requirements of the NHS IGT for Hosted Secondary Use Team/Project, Version 14.1. ${ }^{20}$

\section{Dissemination and public register disclosure}

The outputs from the research will be disseminated primarily through peer review papers in high impact journals within the domains of primary care, surveillance, vaccines and infectious diseases. ${ }^{27},{ }^{28}$ We will present findings at relevant seminars and conferences. The University of Surrey, in accordance with PHE policy, will post a summary of the study protocol and results within 12 months of study completion.

\section{DISCUSSION}

\section{Strengths}

The strengths of this application are that it uses an established sentinel network and builds on PHE expertise in serological analysis to establish a serology bank.

The RCGP RSC is a sentinel network that collects and monitors data from primary care, particularly influenza and other respiratory illnesses, with some practices in the network that have been providing data for decades. ${ }^{11}$ These practices have data quality that is as good as it gets in primary care and the practices are used to taking specimens.

PHE has expertise in serology, but many of serosurveys used residual serum samples from diagnostic blood tests ${ }^{4}$ and hence the clinical information available with these is very limited. Detailed serology surveys are also conducted, but these are extremely expensive.

In this pilot the serology sampling has been designed to provide almost real time results which can be linked to pseudonymised patient data extracted by RCGP RSC. Conducting the sampling alongside routine blood tests reduces overall time for practices and costs overall.

Collection from the same household may result in selection bias as it is likely that they will have had similar exposures; however, it is also a possible strength of the network in trying to understand more about transmission within households or communal establishments, such as old peoples' homes.

We will create a database with individual level serology, virology specimens (if any) confirming influenza or other respiratory disease diagnoses, details of past medical history and vaccine exposure data. 


\section{Limitations}

This pilot is limited by its design as a pilot within the available resource envelope and data quality. The limitations of this study are: its small size, limitation to adult specimens only, no specific targeting of those who have had previous virology or at specific points within the annual cycle of vaccination or of influenza infection and data quality particularly of out-of-practice vaccine exposure.

We set an arbitrary collection strategy across adult age bands. However, this distribution is not representative of our practices populations' age distribution. Any fullscale study would set out to represent the age-sex profile of the population and its geographical distribution.

The study is not powered to detect differences between groups, instead to demonstrate our ability to collect samples across all age groups. The ability to collect across all age groups have been questioned - both by the original authors of the call for a World Serology Bank, ${ }^{29}$ and set as a principal challenge by potential funders if the pilot is a success. Hence the focus of the pilot is on sample collection across age groups.

We have not included children or young people under 18 years in this pilot. We feel this is appropriate for the first pilot of this type. In a substantive study, this group may be important because children are important vectors of disease $\mathrm{e}^{30}$ and the UK is one of the few countries to systematically immunise the population using live attenuated intranasal vaccine (LAIV). ${ }^{31}$

This pilot is not targeting those who have had virology specimens, nor to be aligned with a particular point in the vaccination cycle. Generally vaccination takes place in the early autumn in the UK, with seasonal influenza starting to circulate around the year end. In a major study it may be possible to look at immunity in the population, exposed and unexposed to vaccine, and at residual immunity to the circulating strain of influenza.

Patients vaccinated against influenza outside of General Practice may not have information coded into their computerised medical record system at General Practice. ${ }^{32}$ Vaccination data are sometimes missing, or sometimes incomplete as the standard reporting form from pharmacist to practice only indicate that the person has been vaccinated against influenza, not which brand of vaccine or batch.

\section{CONCLUSION}

This pilot tests our ability to collect samples across all adult age bands within a sentinel network. If successful we will pursue resources to expand this into a larger study.

Acknowledgements Patients for allowing their data to be used for surveillance and research. Practices who have agreed to be part of the RCGP RSC and have allowed us to extract and use health data for surveillance and research. Other members of the Clinical Informatics and Health Outcomes Research Group at University of Surrey. Apollo Medical Systems for data extraction. Collaboration with EMIS, TPP, In-Practice and Micro-test CMR supplier for facilitating data extraction. Patient advisers at practices and colleagues at Public Health England.
Contributors SdeL: is the lead author who developed the study design and wrote the first draft, edited and contributed to subsequent versions of this protocol. MT: liaised with the participating practices, edited and contributed to the writing. RB, $\mathrm{EL}$ and $\mathrm{KH}$ helped develop methods, edited and contributed to subsequent versions of the protocol. FF: manages this study and extensively reviewed the protocol, and contributed to the writing. MZ: reviewed the protocol and contributed to the writing. NA: reviewed the protocol and contributed to the writing. IY: helped develop the practice recruitment methods, edited and contributed to the writing. $\mathrm{MH}$ : helped develop the practice recruitment methods, edited and contributed to the writing. IR: reviewed the protocol and contributed to the writing. RP: helped develop methods and contributed to the subsequent versions of the protocol.

Funding This work was supported by the University of Surrey. The funding was obtained as part of the Higher Education Funding Council for England's (HEFCE) Industrial Strategy funding allocated to the University.

Competing interests Simon de Lusignan has received grant funding through University of Surrey from GSK to report vaccine adverse events and attended advisory boards for Sanofi and Seqirus.

Patient consent for publication Not required.

Ethics approval Ethical approval was granted by the Proportionate Review Sub- Committee of the London - Camden \& Kings Cross on 6 February 2018 (REC Reference: 18/L0/0155). This study received approval from Health Research Authority on 7 February 2018.

Provenance and peer review Not commissioned; externally peer reviewed.

Open access This is an open access article distributed in accordance with the Creative Commons Attribution Non Commercial (CC BY-NC 4.0) license, which permits others to distribute, remix, adapt, build upon this work non-commercially, and license their derivative works on different terms, provided the original work is properly cited, appropriate credit is given, any changes made indicated, and the use is non-commercial. See: http://creativecommons.org/licenses/by-nc/4.0/.

\section{REFERENCES}

1. Metcalf CJ, Farrar J, Cutts FT, et al. Use of serological surveys to generate key insights into the changing global landscape of infectious disease. Lancet 2016;388:728-30.

2. Li ZN, Lin SC, Carney PJ, et al. IgM, IgG, and IgA antibody responses to influenza $A(H 1 N 1) p d m 09$ hemagglutinin in infected persons during the first wave of the 2009 pandemic in the United States. Clin Vaccine Immunol 2014;21:1054-60.

3. Laurie KL, Huston P, Riley S, et al. Influenza serological studies to inform public health action: best practices to optimise timing, quality and reporting. Influenza Other Respir Viruses 2013;7:211-24.

4. Hoschler K, Thompson C, Andrews N, et al. Seroprevalence of influenza A(H1N1)pdm09 virus antibody, England, 2010 and 2011. Emerg Infect Dis 2012;18:1894-7.

5. House of Commons Science and Technology Committee. Scientific advice and evidence in emergencies. Third Report of Session 2010-11. London, 2011.

6. Department of Health. Chief Medical Officer's Statistical Legacy Group (CMO-SLG) pandemic influenza preparedness programme. London, 2010.

7. DH Pandemic Influenza Preparedness Team. UK Influenza pandemic preparedness strategy. London: Department of Health, 2011.

8. The Scientific Pandemic Influenza Advisory Committee. Scientific Pandemic Influenza Advisory Committee (SPI-M) modelling summary. London: Department of Health, 2013.

9. Fineberg HV. Pandemic preparedness and response--lessons from the H1N1 influenza of 2009. N Engl J Med 2014;370:1335-42.

10. de Lusignan S, Correa A, Smith GE, et al. RCGP Research and Surveillance Centre: 50 years' surveillance of influenza, infections, and respiratory conditions. Br J Gen Pract 2017;67:440-1.

11. Correa A, Hinton W, McGovern A, et al. Royal College of General Practitioners Research and Surveillance Centre (RCGP RSC) sentinel network: a cohort profile. BMJ Open 2016;6:e011092.

12. de Lusignan S, Correa A, Pathirannehelage S, et al. RCGP Research and Surveillance Centre Annual Report 2014-2015: disparities in presentations to primary care. Br J Gen Pract 2017;67:e29-40.

13. Pebody R, Warburton F, Ellis J, et al. Effectiveness of seasonal influenza vaccine for adults and children in preventing laboratoryconfirmed influenza in primary care in the United Kingdom: 2015/16 end-of-season results. Euro Surveill 2016;21.

14. Pathirannehelage S, Kumarapeli P, Byford R, et al. Uptake of a dashboard designed to give realtime feedback to a sentinel network 
about key data required for influenza vaccine effectiveness studies. Stud Health Technol Inform 2018;247:161-5.

15. de Lusignan S, Correa A. Opportunities and challenges of a World Serum Bank. Lancet 2017;389:250-1.

16. de Lusignan S. Codes, classifications, terminologies and nomenclatures: definition, development and application in practice. Inform Prim Care 2005;13:65-9.

17. Hoschler K, Andrews NJ, Faust SN, et al. Administration of AS03B-adjuvanted $\mathrm{A}(\mathrm{H} 1 \mathrm{~N} 1)$ pdm09 vaccine in children aged $<3$ years enhances antibody response to $\mathrm{H} 3$ and $B$ viruses following a single dose of trivalent vaccine one year later. Clin Infect Dis 2014;58:181-7.

18. Höschler K, Southern J, Thompson C, et al. Responses to live attenuated influenza vaccine in children vaccinated previously with Pandemrix (ASO3b $>\mathrm{Bb}>$ adjuvanted pandemic $\mathrm{A} / \mathrm{H} 1 \mathrm{~N} 1 \mathrm{pdm09}$ ). Vaccine 2018;36:3034-40.

19. de Lusignan S, Metsemakers JF, Houwink P, et al. Routinely collected general practice data: goldmines for research? A report of the European Federation for Medical Informatics Primary Care Informatics Working Group (EFMI PCIWG) from MIE2006, Maastricht, The Netherlands. Inform Prim Care 2006;14:203-9.

20. Kousoulis AA, Rafi I, de Lusignan S. The CPRD and the RCGP: building on research success by enhancing benefits for patients and practices. Br J Gen Pract 2015;65:54-5.

21. De Meyer F, De Moor G, Reed-Fourquet L. Privacy protection through pseudonymisation in eHealth. Stud Health Technol Inform 2008;141:111-8.

22. de Lusignan S. Effective pseudonymisation and explicit statements of public interest to ensure the benefits of sharing health data for research, quality improvement and health service management outweigh the risks. Inform Prim Care 2014;21:61-3.

23. NHS Digital. Information Governance Toolkit (IGT). https://www.igt. hscic.gov.uk/ (Accessed 16 May 2018).
24. Human Tissue Authority (HTA). List of materials considered to be 'relevant material' under the Human Tissue Act. 2004 https:// www.hta.gov.uk/policies/list-materials-considered-be-\%E2\%80\% 98relevant-material\%E2\%80\%99-under-human-tissue-act-2004 (Accessed 16 May 2018).

25. Mascalzoni D, Dove ES, Rubinstein Y, et al. International charter of principles for sharing bio-specimens and data. Eur J Hum Genet 2015;23:721-8.

26. Clinical Informatics and Health Outcomes Research Group. www. clininf.eu (Accessed 16 May 2018).

27. Medical Research Council. MRC Policy and guidance on sharing of research data from population and patient studies. $2011 \mathrm{https} / / \mathrm{mrc}$. ukri.org/publications/browse/mrc-policy-and-guidance-on-sharingof-research-data-from-population-and-patient-studies/ (Accessed 16 May 2018).

28. Economic Co-operation and Development (OECD). Promoting access to public research data for scientific, economic and social development. 2007 http://www.oecd.org/sti/sci-tech/38500813.pdf (Accessed 16 May 2018).

29. Metcalf CJ, Mina MJ, Winter AK, et al. Opportunities and challenges of a World Serum Bank - Authors' reply. Lancet 2017;389:252.

30. Gerlier L, Lamotte M, Dos Santos Mendes S, et al. Estimates of the public health impact of a pediatric vaccination program using an intranasal tetravalent live-attenuated influenza vaccine in Belgium. Paediatr Drugs 2016;18:303-18.

31. Pebody RG, Green HK, Andrews N, et al. Uptake and impact of vaccinating school age children against influenza during a season with circulation of drifted influenza A and B strains, England, 2014/15. Euro Surveill 2015;20(39.

32. de Lusignan S, Hoghton M, Rafi I. Flu vaccination by pharmacists leads to suboptimal medical records. BMJ 2017;359:j5084. 